\title{
D-29 PREDICTIVE MODELLING OF FRACTURED RESERVOIRS USING GEOMECHANICS AND FLOW SIMULATION
}

S.J. BOURNE, A. RIJKELS, B.J. STEPHENSON and E.J.M. WILLEMSE

Shell International Exploration and Production, P.O. Box 60, 2280 AB Rijswijk, The Netherlands

\begin{abstract}
To optimise recovery in naturally fractured reservoirs, the field-scale distribution of fracture properties must be understood and quantified. We present a semi-deterministic method to systematically predict the spatial distribution of natural fractures and their effect on recovery. The calculated field-scale fracture models are calibrated by geological, well test and field production data to constrain the distributions of fractures within the inter-well space.
\end{abstract}

First, we calculate the stress distribution at the time of fracturing using the present-day structural reservoir geometry. This calculation is based on geomechanical models of rock deformation such as elastic faulting. Second, the calculated stress field is used to govern the simulated growth of fracture networks. Finally, the fractures are upscaled dynamically by simulating flow through the discrete fracture network per grid block, enabling field-scale multi-phase reservoir simulation. Uncertainties associated with these predictions are considerably reduced by constraining and validating the models with seismic, borehole, well test and production data.

This approach is able to predict physically and geologically realistic fracture networks. Its successful application to outcrops and reservoirs demonstrates there is a high degree of predictability in the properties of natural fracture networks. In cases of limited data - where stochastic models typically fail - this method remains robust.

\section{Introduction}

Natural fracture systems have a dramatic impact on reservoir performance, as they act as highly complex and anisotropic flow conduits. Successful management of naturally fractured reservoirs is impossible without substantial knowledge of the properties of the fracture systems on the inter-well scale. This paper presents a new method to predict field-scale natural fracture distributions and their effect on oil and gas recovery (Figure 1). By integrating fracture prediction and flow simulation, we constrain a single field-scale model using all the available geological and flow data. Such improved fracture models improve field development through: (i) better assessment of the recovery mechanism, (ii) more reliable production forecasts, (iii) well placement for optimal drainage, (iv) minimal water-cut, and (v) recognition of drilling hazards associated with fractures.

The following sections describe how structural geometry is used to predict stress, how stress is used to predict fractures, and how fractures are used to predict flow. We finish by presenting one example application of this method to a producing carbonate reservoir. 


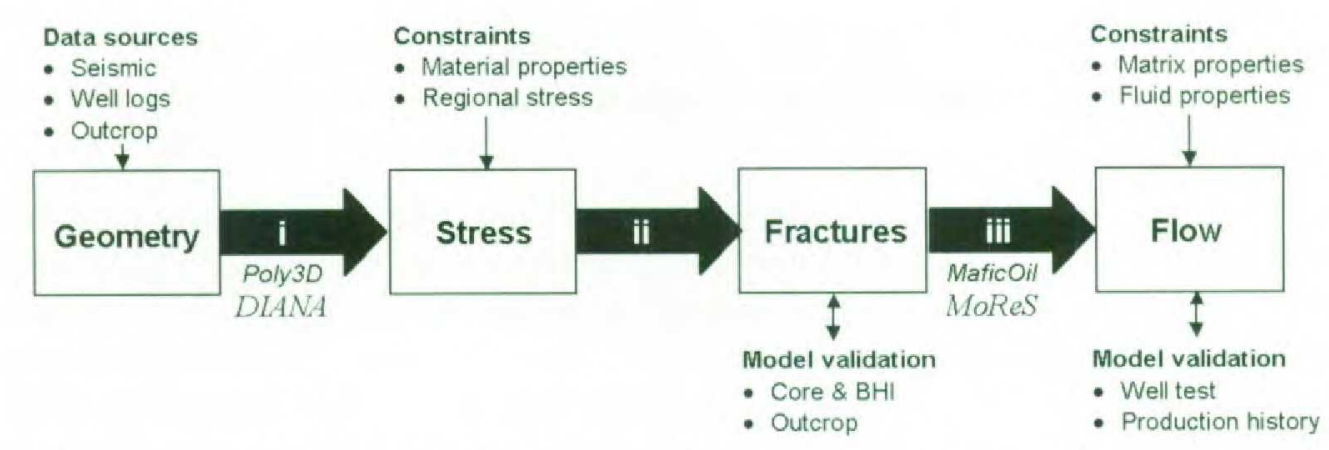

Figure 1. Integrated model for naturally fractured reservoirs based on: (i) geomechanical models of rock deformation, (ii) fracture mechanics, and (iii) multi-phase flow simulation. This workflow incorporates all the available static and dynamic data in order to constrain the model and minimise uncertainty in fracture prediction and flow forecasting.

\section{Stress and fracture prediction}

Faulting is typically the primary deformation mechanism of a reservoir and is therefore expected to be the dominant control on the distribution of fracture networks. The simplest approach to determine the stress field within a faulted reservoir is to assume the rock behaves as a homogeneous, isotropic, and linear-elastic material. The faults are modelled as traction-free surfaces in three dimensions, whose slip in response to a remote stress determines the deformation. We obtain numerical solutions for fault slip and the corresponding stress field using Poly3D [1], a boundary element analysis based on the method of Comninou \& Dunders [2]. Poly3D uses a large number of triangles to represent the complicated three-dimensional fault geometries found in seismic data. Figure 2 illustrates the calculated distribution of elastic stress around a simple hypothetical network of right-lateral strike-slip faults. Even this relatively simple fault geometry produces a complex stress field indicating that fault geometries can dominate the distribution of stress.
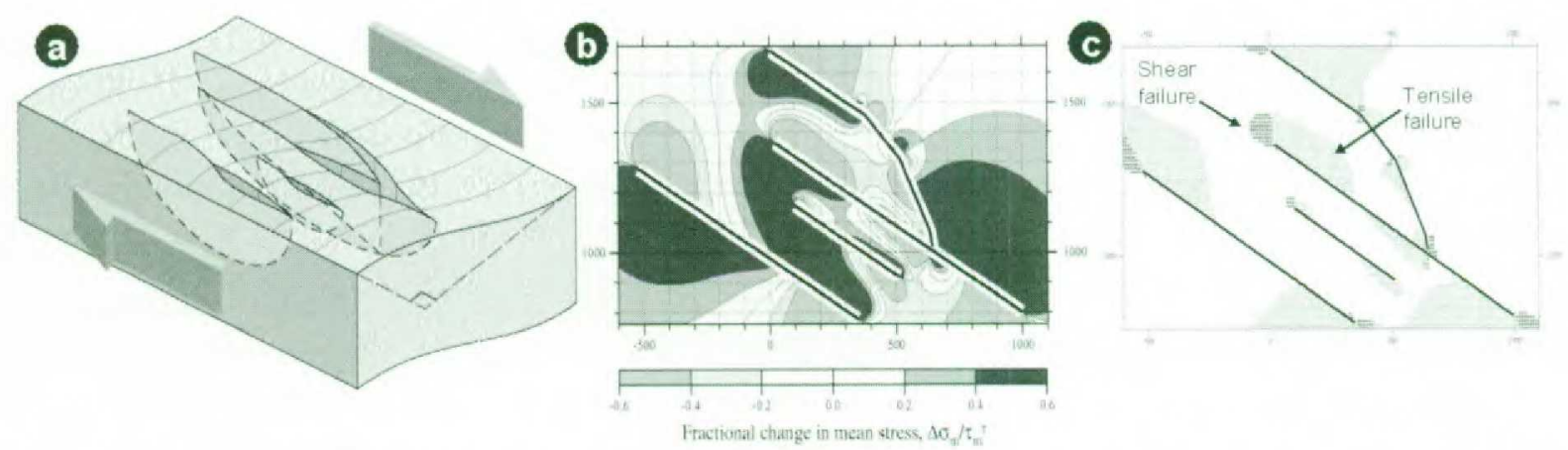

Figure 2. Example of an elastic stress field calculated for a three-dimensional network of rightlateral strike-slip faults. The faults are embedded in a linear elastic, isotropic, homogeneous rock body which is subject to a remote stress (a). The resulting stress field (b) governs the distribution of small-scale tensile and shear fractures shown in (c).

We predict fracturing related to this stress field according to the Griffith stress criterion for tensile failure [3], and a Coulomb stress criterion for shear failure [4]. Whichever criterion is met first as the stress field changes during faulting determines the mode of first failure. The overall shapes of fracture clusters correspond to contours of the elastic stress field, which depend only on fault geometry and the orientation of the remote stress. The lateral extent of fractured areas depends on rock strength relative to the remote stress: increased rock strength results in fractured areas shrinking towards the faults, and vice versa. We include fracture formation mechanisms 
such as fluid pressure increase, diagenesis, cooling, and erosion of the overburden as isotropic reductions in compressive stress. The parameters in the brittle failure analysis can be constrained and the model validated by a small number of wells distributed across the field.

Once the distribution and mode of brittle failure has been determined the actual geometry of fracture networks can be simulated by growing fractures in the brittle-elastic stress field. Fractures initiate in areas of brittle failure, propagate within mechanical layers orthogonal to the direction of least compressive stress, and terminate before entering regions with stable stress states. Fracture spacing and interaction is controlled by a forbidden zone around each fracture which represents an overall reduction in local stress due to the presence of the fracture. Furthermore, when propagating fracture tips approach each other, their mechanical interaction may lead to the fractures turning towards each other and connecting [5]. This is an important mechanism affecting fluid flow as it produces connectivity between essentially parallel fractures.

\section{Flow simulation}

To simulate oil and gas recovery from a predicted fracture network, it must be upscaled to a dual-permeability grid. Dynamic upscaling is possible with MaficOil [6], a finite-element code which simulates single-phase flow through the discrete fracture network inside each grid block, and computes its equivalent fracture permeability tensor. MaficOil also determines the effective fracture spacing, which is used by MoReS (Shell's proprietary dual-permeability simulator [7]) to accurately describe the complicated multi-phase recovery processes in fractured reservoirs. The upscaled fracture grid is then used to obtain a history match on a field-scale, and also to forecast development scenarios.

\section{Application to a producing carbonate reservoir}

This method has been applied successfully to both single- and multi-phase fields. As an illustration, we present results from a fractured oil reservoir in a tight carbonate.

The fracture model predicted for this field is consistent with both fracture orientations and relative intensities as measured from image logs (UBI and CBIL) for 14 wells. Simulations based on the predicted effective fracture permeability grid (Figure 3) matched the production history from 9 of the 10 producing wells. In the simulation, wells were constrained by historical flowing bottom hole pressure data, whilst stimulations and mechanical integrity problems were modelled as a skin change.

All production plots in Figure 3 have the same vertical scale. The plots show two classes of wells: (i) wells that tap fracture clusters with high rates (top row) and an ultimate recovery (UR) that is effectively determined by fracture cluster size; and (ii) wells located in unfractured regions with low rates (bottom row), whose UR is determined by matrix properties alone. The middle row shows wells with integrity problems or stimulated wells.

Our geomechanical fracture model matches the (decline) rates in 9 wells, and pressure readings in 6 observation wells. This match is obtained using a single field-scale model without any local adjustments. Sensitivity studies show that the model is constrained very tightly. Well recompletions have confirmed the predictive capabilities of this fracture model, and production rates from all wells (including those recompleted) are, to date, consistent with the flow forecast from the fracture model. The ability to predict fracture distribution and hydraulic properties on a field-scale has a clear business impact, as it increases the probability of success of a new well significantly. 

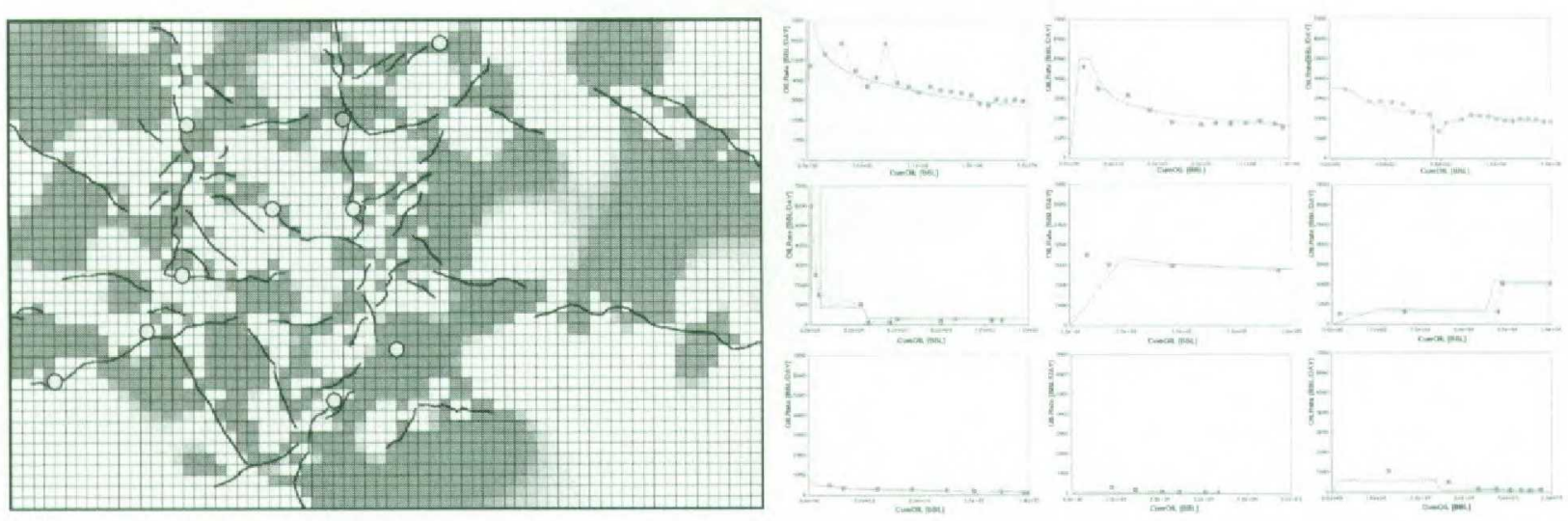

Figure 3. Left: Map showing effective fracture permeability around seismically imaged faults (black lines) based on dynamic upscaling of a predicted, discrete fracture network. Grid block dimensions are $200 \mathrm{~m}$ by $200 \mathrm{~m}$. Right: History match for the wells shown in the map as circles. Each graph shows the production rate versus cumulative oil produced. Actual production history is denoted by black squares and the simulation result by the other line.

\section{Conclusions}

In short, rocks obey physics, and physics can be used to predict fractures that affect flow across entire naturally fractured reservoirs. As our model parameters are field-scale, information from each well constrains the whole fracture model and not just the areas close to wells. This makes the model suitable for fracture prediction and flow forecasting in all parts of the reservoir. Traditional geostatistical methods of fracture modelling are different to the model described above. They rely on stochastic realisations of the large numbers of fracture networks consistent with borehole fracture data to explain inflow data. This approach is ultimately limited to nearwell scales as it lacks information on how fracture statistics change away from wells.

In contrast, access to a predictive field-scale fracture model allows enhanced field development of naturally fractured reservoirs. Understanding and quantification of the fracture system helps to mitigate risks via improved flow forecasting, and optimal well placement and design.

\section{References}

[1] Thomas, A.L. 1993. Poly3D: A three-dimensional, polygonal element, displacement discontinuity boundary element computer program with applications to fractures, faults, and cavities in the Earth's crust. Masters dissertation, Stanford University, California 94305-2115.

[2] Comninou, M.A., Dunders, J. 1975. The angular dislocation in a half-space. J. Elast., 5,216.

[3] Griffith, A.A. 1921. The phenomena of rupture and flow in solids. Phil. Trans. Roy. Soc., London, A 221, 163-198.

[4] Coulomb, C.A. 1773. Sur une application des règles de Maximis et Minimis a quelques problèmes de stratique relatifs à l'Architecture, Acad. Roy. Des Sciences Memoires de math. Et de physique par divers savans, $7,343-382$.

[5] Olson, J.E., D.D. Pollard 1990. The initiation and growth of en échelon veins. J. Struc Geol., 13, 5, 595-608. See also: Cruikshank, K.M. G. Zhao, A.M. Johnson 1991. Analysis of minor fractures associated with joints and faulted joints. J. Struc. Geol., 13, 8, 865-886.

[6] MaficOil, 1998. An special purpose version of Mafic. See: www.golder.com for a description

[7] Boerrigter, P.M., L.E.C. van de Leemput, J., Pieters, K., Wit, J.G.J. Ypma, 1993. Simulation of fractured reservoirs - case studies. SPE 26515. 